\title{
Tippe Top Inversion as a Dissipation-Induced Instability*
}

\author{
Nawaf M. Bou-Rabee ${ }^{\dagger}$, Jerrold E. Marsden ${ }^{\ddagger}$, and Louis A. Romero§
}

Abstract. By treating tippe top inversion as a dissipation-induced instability, we explain tippe top inversion through a system we call the modified Maxwell-Bloch equations. We revisit previous work done on this problem and follow Or's mathematical model [SIAM J. Appl. Math., 54 (1994), pp. 597-609]. A linear analysis of the equations of motion reveals that the only equilibrium points correspond to the inverted and noninverted states of the tippe top and that the modified Maxwell-Bloch equations describe the linear/spectral stability of these equilibria. We supply explicit criteria for the spectral stability of these states. A nonlinear global analysis based on energetics yields explicit criteria for the existence of a heteroclinic connection between the noninverted and inverted states of the tippe top. This criteria for the existence of a heteroclinic connection turns out to agree with the criteria for spectral stability of the inverted and noninverted states. Throughout the work we support the analysis with numerical evidence and include simulations to illustrate the nonlinear dynamics of the tippe top.

Key words. tippe top inversion, dissipation-induced instability, constrained rotational motion, axisymmetric rigid body

AMS subject classifications. 70E18, 34D23, 37J15, 37M05

DOI. $10.1137 / 030601351$

1. Introduction. Tippe tops come in a variety of forms. The most common geometric form is a cylindrical stem attached to a truncated ball, as shown in Figure 1.1. On a flat surface, the tippe top will rest stably with its stem up. However, spun fast enough on its blunt end, the tippe top momentarily defies gravity, inverts, and spins on its stem until dissipation causes it to fall over. This spectacular sequence of events occurs because, and in spite of, dissipation.

Tippe top inversion is an excellent example of a dissipation-induced instability. Tippe top inversion is described by a system we call the modified Maxwell-Bloch equations. These

${ }^{*}$ Received by the editors October 22, 2003; accepted for publication (in revised form) by M. Golubitsky January 22, 2004; published electronically July 6, 2004. This work was performed by an employee of the U.S. Government or under U.S. Government contract. The U.S. Government retains a nonexclusive, royalty-free license to publish or reproduce the published form of this contribution, or allow others to do so, for U.S. Government purposes. Copyright is owned by SIAM to the extent not limited by these rights.

http://www.siam.org/journals/siads/3-3/60135.html

${ }^{\dagger}$ Applied and Computational Mathematics, Caltech, Pasadena, CA 91125 (nawaf@acm.caltech.edu). The research of this author was supported by the U.S. DOE Computational Science Graduate Fellowship through grant DE-FG0297ER25308.

${ }^{\ddagger}$ Control and Dynamical Systems, Caltech, Pasadena, CA 91125 (marsden@cds.caltech.edu). The research of this author was partially supported by the National Science Foundation.

§Sandia National Laboratories, P.O. Box 5800, MS 1110, Albuquerque, NM 87185-1110 (Iromero@sandia.gov). The research of this author was supported by Sandia National Laboratories. Sandia is a multiprogram laboratory operated by Sandia Corporation, a Lockheed Martin Company, for the United States Department of Energy under contract DE-AC04-94AL85000. 

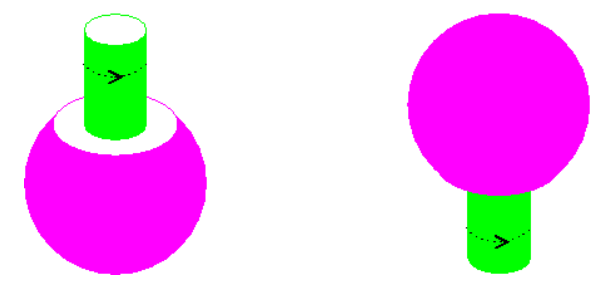

Figure 1.1. From left: a sketch of the noninverted and inverted states of the tippe top.

equations are a generalization of a previously derived normal form describing dissipationinduced instabilities in the neighborhood of the 1:1 resonance [4]. We will show in section 2 that the modified Maxwell-Bloch equations are the normal form for rotationally symmetric, planar dynamical systems.

A dissipation-induced instability describes a neutrally stable equilibrium becoming spectrally (and hence nonlinearly) unstable with the addition of dissipation. Dissipation-induced instability itself has a long history, which goes back to Thomson and Tait; see [14]. In its modern form, dissipation-induced instability was shown both to be a general phenomenon for gyroscopically stabilized systems and to provide a sharp converse to the energy momentum stability method by Bloch et al. in [1] and [2]. We refer the reader to these papers for additional examples and basic theory.

By considering tippe top inversion as a dissipation-induced instability, we observe precisely how tippe top inversion relates to well-understood dissipation-induced instabilities in gyroscopic systems.

History and literature. Tippe top inversion has been much investigated in the literature but never satisfactorily resolved. In what follows we survey a selection of theoretical results relevant to this paper. We refer the reader to the work of Cohen [5] and Or [11] for more comprehensive surveys of the literature.

Analysis of the tippe top dates back to the last century with Routh's commentary on the rising of tops. Routh's simple physical analysis made it clear that dissipation was fundamental in understanding the physics of tippe top inversion [12]. In 1977, Cohen confirmed Routh's physical analysis through numerical simulation [5]. Cohen modeled the tippe top as a holonomic ball with an inhomogeneous, axisymmetric mass distribution on a fixed plane. Thus, the ball's center of mass was on its axis of symmetry, but not coincident with its geometric center. Tippe top inversion corresponded to the ball's center of gravity moving above its geometric center. Cohen's mathematical model, derived using Newtonian mechanics, is written in terms of Euler angles and assumed a sliding friction law.

In reality, tippe top inversion is a transient phenomenon because of dissipation. However, by neglecting frictional torque, the spinning inverted state becomes a steady-state phenomenon. With a sliding friction law that neglects frictional torque, Cohen's equations of motion were amenable to a linear-stability analysis about the spinning inverted and noninverted states, but he did not carry out such an analysis in his work. In this sense the fact that the model neglects frictional torque is a feature rather than a defect of the friction law.

Cohen concluded that a sliding friction law based on Coulomb friction explains tippe top 
inversion. Moreover, Cohen showed that the inviscid ball (no sliding friction) does not invert. Coulomb friction is proportional to the normal force exerted by the surface at the point of contact and opposes the motion of the tippe top at the point of contact. This nonlinear friction law drops out of a standard linear-stability analysis.

In 1994, Or extended Cohen's work by generalizing his friction law, performing a dimensional analysis of the equations of motion, writing the equations of motion in convenient coordinates, and exploring the linearized behavior of the ball. To further facilitate a linearstability analysis, Or added viscous friction to Cohen's friction law. The viscous friction is linearly related to the slip velocity at the point of contact.

Or's work showed that inversion could occur because of viscous or Coulomb friction. Or's analysis of tippe top inversion by Coulomb friction and viscous friction demonstrated viscous friction flips the top more rapidly than Coulomb friction. His analysis mentioned first integrals in the no-slip problem, but did not use them to explain the global behavior of the tippe top.

There was good reason to avoid a global analysis of the tippe top: the theoretical model of a ball did not accurately model the contact effects of the tippe top stem. However, the fundamental physics behind the global behavior of the tippe top was still reasonably described by the equations of motion for a ball with an eccentric center of mass, i.e., a gyroscopic, axisymmetric rigid body rising from a gravitationally favorable position to an unfavorable one because of dissipation. This observation motivated some aspects of the present study and possibly Ebenfeld's thesis on tippe top inversion from a Lyapunov perspective.

In 1995, Ebenfeld and Scheck applied a Lyapunov method to a dimensional version of Or's mathematical model. Using the energy as a Lyapunov function, they analyzed the orbital stability of the spinning, sliding ball. They showed that, starting with nonzero slip, the standing, spinning tippe top tends to manifolds of constant energy with no-slip and no tangential surface force. Moreover, they showed that without slip the tippe top cannot invert. They classified all possible asymptotic solutions as either tumbling (precession, spin, and no nutation) or rotating (pure spin) solutions. They provide criteria for determining the Lyapunov stability of these solutions [6].

We have applied some techniques of the present work to the related, but different, "rising egg" problem. (See Bou-Rabee, Marsden, and Romero [3].) In that paper we address the contributions of Moffatt [10] and Ruina [13] to that problem.

Main goals of this paper. The main result of this paper asserts that the stability of the noninverted and inverted tippe top (cf. Figure 1.1) and the existence of a heteroclinic connection between these states is completely described by the modified Maxwell-Bloch equations: a normal form for rotationally symmetric, planar dynamical systems. This result is important because (1) it shows how tippe top inversion relates to well-studied dissipation-induced instabilities in gyroscopic systems; (2) it integrates the linear and nonlinear analysis; and (3) it simplifies the analysis of tippe top inversion and yields explicit criteria for when the tippe top inverts.

We use Or's mathematical model with viscous friction to obtain equations of motion for the tippe top in a convenient set of coordinates. We locate and analyze the stability of all equilibrium points of the equations of motion. As Ebenfeld observed, the viscous problem conserves $\Upsilon_{Q}$, the angular momentum about the vector connecting the center of mass of the 
ball to the surface point of contact. For a fixed value of $\Upsilon_{Q}$, the only equilibrium points of the equations of motion correspond to the noninverted and inverted states of the tippe top. We extend Or's linear analysis by linearizing about both of these equilibrium points.

By assuming that the translation of the center of mass is negligible, we derive a reduced system for the tippe top in terms of only angular variables. We show that this reduced equation is of the form of a modified Maxwell-Bloch equation and give reasons to support this approximation. Analysis of explicit stability criteria for the reduced system shows that the reduced system accurately describes the linearized behavior of the tippe top. Thus, the paper shows that the stability of the noninverted and inverted tippe top is completely determined by the modified Maxwell-Bloch equations. Moreover, these equations are the normal form for tippe top inversion; i.e., they are the simplest possible equations that can capture tippe top inversion.

We extend the nonlinear analysis by proving the existence of a heteroclinic connection between the noninverted and inverted states of the tippe top. Application of LaSalle's invariance principle repeats/simplifies Ebenfeld's energy arguments. Like Ebenfeld, we use the energy as a Lyapunov function. For the viscous problem, we show that the energy's orbital derivative is negative semidefinite and a constant multiple of the $\ell_{2}$-norm of the slip velocity. Thus, when the slip velocity vanishes, the energy is conserved. We identify the largest invariant set on this manifold characterized by no-slip and no tangential surface force.

The largest invariant set on this manifold extremizes the energy subject to the surface and angular momentum constraint: $\Upsilon_{Q}=$ constant. For certain parameter values, we find that the noninverted and inverted equilibrium states are the only extrema of the energy and are, in fact, the absolute maxima and minima of the energy, respectively. By the theorems of Barbashin and Krasovskii, trajectories starting in the neighborhood of the asymptotically unstable, noninverted state approach the asymptotically stable, inverted state as $t \rightarrow \infty$; i.e., the inverted state is globally asymptotically stable [8]. Outside this range of parameter values, the inverted and noninverted states become asymptotically unstable and a limit cycle corresponding to Ebenfeld's tumbling solution minimizes the energy.

Thus, the asymptotic states of the tippe top approach either (i) an isolated asymptotically stable equilibrium point or (ii) a neutrally stable limit cycle. The second case corresponds to the inverted and noninverted states being asymptotically unstable. In this case trajectories starting in the neighborhood of the noninverted, asymptotically unstable state will tend to limit cycles upon which total energy, magnitude of the angular momentum, nutation angle, and angular momentum about the vertical are conserved. We refer the reader to Ebenfeld for conditions for Lyapunov stability of this limit cycle. When the inverted state is asymptotically stable, a heteroclinic connection between the asymptotically unstable and stable states of the tippe top exists. We derive explicit criteria to determine the range of parameter values for which the heteroclinic connection exists.

A comparison of the existence criteria for the heteroclinic connection and the linearstability criteria derived from the modified Maxwell-Bloch system for the tippe top shows they are explicitly related. Thus, the modified Maxwell-Bloch equations fully explain tippe top inversion.

Without dissipation, linear theory concludes that the equilibria are linearly (or neutrally) stable, which does not imply nonlinear stability. With dissipation, however, it is no surprise that the linear analysis provides the correct local nonlinear dynamics (since dissipation moves 
eigenvalues off of the imaginary axis).

Organization of the paper. In section 2, we present the modified Maxwell-Bloch equations. We derive these equations and supply specific stability criteria for the system's characteristic polynomial. We also discuss the ability of this model to capture the fundamental physics in tippe top inversion.

In section 3, we derive and discuss the mathematical model of the tippe top. Specifically, we write the dimensional equations of motion of the theoretical tippe top using Newtonian mechanics, discuss the friction law, and nondimensionalize these equations. We also explicitly compare the model in this paper to others in the literature.

In section 4, we apply linear theory to the nonlinear model. In particular, we locate the equilibria of the governing dimensionless equations, linearize about these states, as well as review and extend Or's numerical stability analysis.

In section 5 , we cast the linearized equations for the tippe top in the form of the modified Maxwell-Bloch equations. We mention a direct derivation to these equations and analyze the stability of the modified Maxwell-Bloch equations. In particular, we show that these equations are the simplest possible equations that can capture the fundamental physics in tippe top inversion.

In section 6, we explain tippe top inversion from an energy landscape perspective. We apply LaSalle's invariance principle to determine the asymptotic state of the tippe top, study the behavior of solutions on this asymptotic state, and determine when this asymptotic state corresponds to the inverted tippe top. We also compare the criteria for the existence of a heteroclinic connection with the linear-stability criteria for the tippe top modified MaxwellBloch equations provided in section 5 .

In section 7 , we discuss data from simulations which verify the local and global analysis in the paper. We conclude the paper with a discussion of future directions inspired by this work.

2. Modified Maxwell-Bloch equations. This section introduces an important extension of the Maxwell-Bloch equations and studies their stability.

Derivation. Consider a planar ODE of the form

$$
\ddot{q}=f(q, \dot{q}), \quad q=\left[\begin{array}{l}
x \\
y
\end{array}\right] .
$$

Linearization of these equations yields

$$
\ddot{q}=A \dot{q}+B q .
$$

The characteristic polynomial of this system

$$
\operatorname{det}\left(\left[\begin{array}{cc}
\sigma^{2} & 0 \\
0 & \sigma^{2}
\end{array}\right]+A \sigma+B\right)=0
$$

shows that the ODE will have time-reversal symmetry; i.e., if $\sigma$ is a solution, then so is $-\sigma$, when $A$ is skew-symmetric and $B$ is symmetric. 
We define the rotation matrix

$$
R(\theta)=\left[\begin{array}{cc}
\cos (\theta) & -\sin (\theta) \\
\sin (\theta) & \cos (\theta)
\end{array}\right]
$$

as well as the identity and elementary skew-symmetric matrix in $\mathbb{R}^{2}$ :

$$
I=\left[\begin{array}{ll}
1 & 0 \\
0 & 1
\end{array}\right], \quad S=\left[\begin{array}{cc}
0 & -1 \\
1 & 0
\end{array}\right]
$$

The necessary and sufficient condition for a $2 \times 2$ matrix in $\mathbb{R}^{2}$ to commute with the rotation matrix is that the matrix be a linear combination of $I$ and $S$.

Thus, if this ODE is rotationally symmetric, i.e., the ODE is invariant under $\mathrm{SO}(2)$ rotation, then the matrices $A$ and $B$ can be expressed as

$$
A=-\alpha S-\beta I, \quad B=-\gamma S-\delta I,
$$

where $\alpha, \beta, \gamma$, and $\delta$ are real scalars. Because $\alpha$ and $\gamma$ can destroy time-reversal symmetry in $A$ and $B$, we call these terms dissipative.

Given the particular form of the rotationally symmetric ODE, we can write the twodimensional real system as a one-dimensional complex system,

$$
\ddot{z}+i \alpha \dot{z}+\beta \dot{z}+i \gamma z+\delta z=0, \quad z=x+i y,
$$

which we call the modified Maxwell-Bloch equations. We observe that (2.1) is the basic harmonic oscillator with the two complex terms $i \alpha \dot{z}$ and $i \gamma z$. In physical systems, the first term arises from Coriolis effects, and hence is known as the gyroscopic term. The second term typically arises from dissipation in rotational variables. This damping force is different from the usual damping term proportional to absolute velocity, $\beta \dot{z}$. Physically the complex damping term models viscous effects caused by, for example, motion in a fluid, while the usual damping term models internal dissipation.

Proposition 2.1. The modified Maxwell-Bloch equations are the linearized normal form for planar, rotationally symmetric dynamical systems. is

Stability criteria. The characteristic polynomial of the modified Maxwell-Bloch equations

$$
\lambda^{4}+2 \beta \lambda^{3}+\left(\alpha^{2}+\beta^{2}+2 \delta\right) \lambda^{2}+2(\alpha \gamma+\beta \delta) \lambda+\left(\gamma^{2}+\delta^{2}\right)=0
$$

We now write the necessary and sufficient conditions for this polynomial to be Hurwitz [7].

Proposition 2.2. The zero solution of the modified Maxwell-Bloch equations is asymptotically stable provided that the following inequalities hold:

$$
\begin{aligned}
\beta & >0, \\
\alpha \beta \gamma-\gamma^{2}+\beta^{2} \delta & >0, \\
\alpha^{2} \beta+\beta^{3}-\alpha \gamma+\beta \delta & >0 .
\end{aligned}
$$

There are two especially interesting physical cases of these equations: 
1. When $\delta>0, \gamma=\beta=0$, the system is neutrally stable with or without the presence of the gyroscopic term. Adding usual dissipation $(\beta>0)$ makes the neutrally stable zero solution asymptotically stable. Adding, however, damping in rotational variables can stabilize or destabilize the neutrally stable zero solution.

2. When $\delta<0, \alpha>-4 \delta>0, \beta=\gamma=0$, the system is gyroscopically, and hence neutrally, stable. Adding usual damping makes the neutrally stable zero solution asymptotically unstable since the second inequality in (2.2) can never hold. This case corresponds to the classical dissipation-induced instability [1]. If $\beta=0$ and $\beta>0$, the neutrally stable zero solution becomes asymptotically unstable. Adding damping in both variables, i.e., $\beta>0$ and $\gamma>0$, can stabilize or destabilize the zero solution depending on the ratio of $\beta$ to $\gamma$.

For the tippe top, we will show that dissipation in rotational variables (or complex damping) is essential to understanding inversion. In fact, the remarks above point out some limitations of usual damping: usual damping can only predict instability in the case of a gyroscopically stable system and stability in the case of a gravitationally stable system.

Consider the modified Maxwell-Bloch equations as a possible model of the linearized behavior of the tippe top. In particular, suppose that the noninverted and inverted states of the tippe top correspond to the zero solution of (2.1). In the inviscid case, we observe a noninverted state which is gravitationally stable with or without gyroscopic effects. Remark 1 above shows that the addition of usual damping cannot destabilize this gravitationally stable, noninverted state. The complex damping term, however, can destabilize this state. Therefore, the complex damping term can explain why the gravitationally stable tippe top becomes asymptotically unstable.

Moreover, after the tippe top inverts we have a gyroscopically stabilized inverted state. We have shown that the addition of usual damping would make such a system asymptotically unstable. Thus, usual damping cannot explain why the tippe top spins stably in its inverted state. Remark 2 shows that the complex and usual damping term in the right ratio can, however, stabilize this state. Thus, the complex damping term can also explain why the tippe top spins stably on its stem. We will revisit this analysis when we cast the linearized equations of the tippe top in the form of the modified Maxwell-Bloch equations.

3. Tippe top governing equations. This section contains a pedagogical derivation of the tippe top governing equations following Or [11], given mainly for the reader's convenience. The section concludes with a brief discussion of how to explicitly obtain the equations derived by Or [11] and Ebenfeld and Scheck [6] from our form of the equations and how to formulate the equations of motion using Lagrangian mechanics.

Derivation. We write the equations of motion of the tippe top from first principles. We idealize the tippe top as a ball of radius $R$ and mass $M$ on a fixed plane. The mass distribution of the ball is inhomogeneous, but symmetric about an axis through the ball's geometric center. Thus, the ball's center of mass is located on the axis of symmetry $\mathbf{k}$, which is pointing in the direction $(l, m, n)$, but at a distance $R e^{\star}$ above the geometric center, where $e^{\star}$ is the center of mass offset $\left(0 \leq e^{\star} \leq 1\right)$.

Let the points $Q, O$, and $C$ represent the point of contact, the geometric center, and the center of mass of the ball, respectively (cf. Figure 3.1). We define $\mathbf{e}_{x}, \mathbf{e}_{y}, \mathbf{e}_{z}$ to be unit vectors 
of a nonrotating Cartesian frame attached to $O$. We define principal axes as $\mathbf{i}, \mathbf{j}, \mathbf{k}$ with axis of symmetry $\mathbf{k}$. Let $\mathbf{L}, \boldsymbol{\omega},(X, Y, Z)$ be the ball's angular momentum about $O$, angular velocity, and absolute position of its center of mass, respectively. We define $I_{k}$ and $I=I_{i}=I_{j}$ to be the inertias about the respective principal axes attached to $O$.

Let the vector $\mathbf{Q}$ represent the position of the point of contact $Q$ with respect to the center of mass $O$ given by

$$
\mathbf{Q}=R\left(-e^{\star} \mathbf{k}-\mathbf{e}_{z}\right)
$$

We assume that the only external forces acting on the tippe top are due to gravity and the surface force $\mathbf{F}_{Q}$ at the point of contact $Q$. Conservation of linear momentum yields

$$
\begin{aligned}
M \ddot{X} & =\mathbf{F}_{Q} \cdot \mathbf{e}_{x}, \\
M \ddot{Y} & =\mathbf{F}_{Q} \cdot \mathbf{e}_{y}, \\
M \ddot{Z} & =\mathbf{F}_{Q} \cdot \mathbf{e}_{z}-M g .
\end{aligned}
$$

By the translation theorem of angular momentum, we have

$$
\dot{\mathbf{L}}=M R^{2}\left(e^{\star}\right)^{2} \mathbf{k} \times \ddot{\mathbf{k}}+\mathbf{Q} \times \mathbf{F}_{Q} .
$$

Since the axis of symmetry undergoes pure rotation, we have

$$
\dot{\mathbf{k}}=\omega \times \mathbf{k},
$$

which is known as the attitude equation. By projecting the angular momentum on the principal coordinate frame, we can relate $\boldsymbol{\omega}$ and $\mathbf{L}$ as follows:

$$
\mathbf{L}=I \boldsymbol{\omega}+\frac{I_{k}-I}{I_{k}}(\mathbf{L} \cdot \mathbf{k}) \mathbf{k}
$$

Solving for $\boldsymbol{\omega}$ we obtain

$$
\boldsymbol{\omega}=\frac{1}{I}\left(\mathbf{L}+\frac{I_{k}-I}{I_{k}}(\mathbf{L} \cdot \mathbf{k}) \mathbf{k}\right)
$$

Substituting this expression into the attitude equation, we obtain

$$
\dot{\mathrm{k}}=\frac{1}{I} \mathbf{L} \times \mathbf{k} .
$$

We also have the constraint that keeps the ball on the fixed plane (no hopping or penetration into the surface):

$$
\mathbf{Q} \cdot \mathbf{e}_{z}+z=0
$$

Together, (3.2), (3.3), (3.4), and (3.5) represent the dimensional equations of motion of the ball once the force $\mathbf{F}_{Q}$ has been specified. 


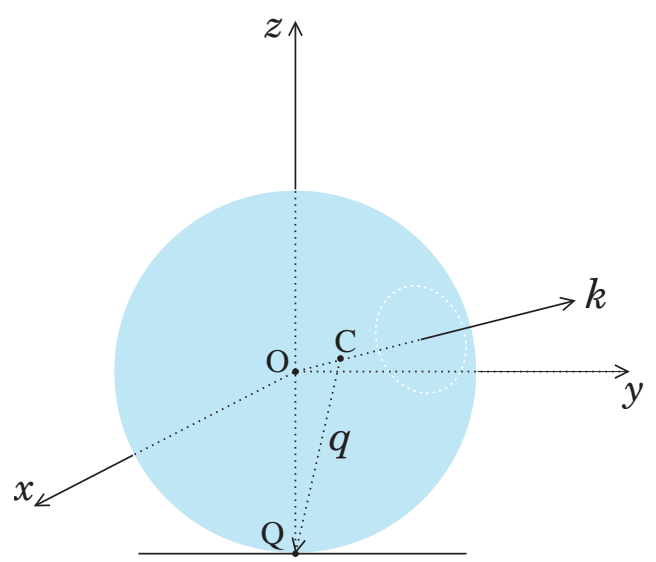

Figure 3.1. We model the tippe top as a ball with an eccentric center of mass $C$, geometric center $O$, and point of contact $Q$. Vectors $\mathbf{q}$ and $\mathbf{k}$ represent the dimensionless position of the contact point with respect to the center of mass and the unit vector in the direction of the axis of symmetry, respectively.

Friction law. The force exerted on the body at the contact point $Q$ is due to surface frictional and normal reaction forces: $\mathbf{F}_{Q}=\mathbf{F}_{f}+F_{z} \mathbf{e}_{z}$. It is well understood that sliding friction is the main mechanism behind tippe top inversion. To facilitate the linear and nonlinear analysis, we assume a sliding frictional force proportional to the slip velocity, i.e., the velocity of the contact point on the rigid body relative to the center of mass $\mathbf{V}_{Q}$ :

$$
\mathbf{F}_{f}=-c \mathbf{V}_{Q}
$$

A more complete friction law would include rotational, pure rolling, and Coulomb friction. We neglect frictional torque so that the spinning, standing ball is a steady-state solution of the equations of motion. Addition of nonlinear Coulomb friction would result in algebraic rather than exponential divergence of the unstable equilibrium solution [11]. Pure rolling friction would be important in the limit to a perfectly rough surface.

The slip velocity, i.e., the velocity of the contact point on the rigid body relative to the center of mass, is

$$
\mathbf{V}_{Q}=\mathbf{V}_{C}+\boldsymbol{\omega} \times \mathbf{Q}
$$

where $\mathbf{V}_{C}=(\dot{X}, \dot{Y}, \dot{Z})$ is the absolute velocity of the center of mass. The slip velocity in terms of the angular momentum is given by

$$
\mathbf{V}_{Q}=\mathbf{V}_{C}+\frac{R}{I}\left(\left(e^{\star} \mathbf{k}+\mathbf{e}_{z}\right) \times \mathbf{L}+\frac{I_{k}-I}{I_{k}}(\mathbf{L} \cdot \mathbf{k})\left(\mathbf{e}_{z} \times \mathbf{k}\right)\right) .
$$

Dimensionless equations. We introduce the following dimensionless variables:

$$
x=\frac{X}{R}, \quad y=\frac{Y}{R}, \quad z=\frac{Z}{R}, \quad t=T \Omega, \quad \mathbf{f}_{Q}=\frac{R \mathbf{F}_{Q}}{I_{k} \Omega^{2}}, \quad \mathbf{\Upsilon}=\frac{\mathbf{L}}{I_{k} \Omega}, \quad \mathbf{q}=\frac{\mathbf{Q}}{R},
$$


and parameters

$$
\sigma=\frac{I_{k}}{I}, \quad \operatorname{Fr}^{-1}=\frac{g}{\Omega^{2} R}, \quad \mu=\frac{M R^{2}}{I}, \quad \nu=\frac{c R^{2}}{I \Omega},
$$

where $\Omega$ is the spin rate of the initially standing equilibrium solution we will linearize about and $T$ represents dimensional time. The dimensionless parameters $\sigma, \operatorname{Fr}, \mu$, and $\nu$ are the inertia ratio, Froude number, dimensionless mass, and friction factor, respectively.

The governing equations follow:

$$
\begin{aligned}
\mu \ddot{x} & =\sigma f_{x}=-\nu\left[\dot{x}-e^{\star} i-\sigma \Upsilon_{y}+(\sigma-1)(\mathbf{\Upsilon} \cdot \mathbf{k}) m\right] \\
\mu \ddot{y} & =\sigma f_{y}=-\nu\left[\dot{y}-e^{\star} \dot{m}+\sigma \Upsilon_{x}-(\sigma-1)(\mathbf{\Upsilon} \cdot \mathbf{k}) l\right], \\
\dot{\mathbf{\Upsilon}} & =\frac{\left[\sigma^{2} \mu\left(e^{\star}\right)^{2}(\mathbf{\Upsilon} \cdot \mathbf{k})(\mathbf{k} \times \mathbf{\Upsilon})+e^{\star} \sigma f_{z}\left(\mathbf{e}_{z} \times \mathbf{k}\right)+\sigma \mathbf{q} \times \mathbf{f}_{f}-\sigma \mu\left(e^{\star}\right)^{2}\left(\mathbf{f}_{f} \cdot\left(\mathbf{k} \times \mathbf{e}_{z}\right)\right) \mathbf{k}\right]}{\left(1-\mu\left(e^{\star}\right)^{2}\right) \sigma}, \\
\dot{\mathbf{k}} & =\sigma \mathbf{\Upsilon} \times \mathbf{k} .
\end{aligned}
$$

Equation (3.7) describes the translational $(x, y)$ and rotational $(\mathbf{\Upsilon}, \mathbf{k})$ motion of the ball.

To determine the normal reaction force $f_{z}$ we use the constraint that keeps the ball on the surface,

$$
z=1+e^{\star}\left(\mathbf{e}_{z} \cdot \mathbf{k}\right)
$$

together with the vertical translation equation,

$$
\ddot{z}=\frac{\sigma}{\mu} f_{z}-\operatorname{Fr}^{-1} \text {. }
$$

The appendix gives an explicit expression for the normal reaction force.

Observe that the angular momentum about the vector connecting the center of mass to the contact point $\mathbf{q}$ is a conserved quantity for (3.7):

$$
\Upsilon_{Q}=\Upsilon_{c g} \cdot \mathbf{q}=-\sigma e^{\star}(\mathbf{\Upsilon} \cdot \mathbf{k})\left(1+\sigma \mu e^{\star} n\right)-\sigma\left(1-\sigma \mu\left(e^{\star}\right)^{2}\right) \Upsilon_{z}, \quad\left(\Upsilon_{Q}\right)_{t}=0
$$

This first integral, known in the literature as the Jellett invariant, can be derived geometrically from a momentum map. The Jellett invariant corresponds to an $\mathbb{S}^{1}$ action on the configuration space $Q=\mathrm{SO}(3) \times \mathbb{R}^{2}$, which can be computed by formula (12.2.1) of [9]. Specifically, the action for $\theta \in \mathbb{S}^{1}$ is given by simultaneous rotation about the axis of symmetry by the angle $-\sigma e^{\star}\left(1+\sigma \mu e^{\star} n\right) \theta$ and about the vertical by the angle $\left(1-\sigma \mu\left(e^{\star}\right)^{2}\right) \theta$. Because the force at the point of contact does no virtual work under this action, dissipation does not destroy this conservation law.

We briefly compare this model with others in the literature. Writing the absolute velocity of the center of mass in terms of the absolute velocity of the center of curvature leads to the form of the equations that can be found in Or's work [11, eqs. (1) and (2), p. 600]:

$$
\begin{aligned}
\mathbf{V}_{0} & =\mathbf{v}_{C}-e^{\star} \sigma \mathbf{\Upsilon} \times \mathbf{k} \\
\boldsymbol{\omega} & =\frac{1}{\sigma}(\boldsymbol{\omega}+(\sigma-1)(\boldsymbol{\omega} \cdot \mathbf{k}) \mathbf{k})
\end{aligned}
$$


We choose to write the governing equations in terms of the velocity of the center of mass because we are interested in the translational effects of the ball's center of mass. Writing the angular momentum with respect to the center of mass $C$ leads to the form of the equations found in Ebenfeld's work [6, eq. (14), p. 201]:

$$
\Upsilon_{c g}=\left(1-\sigma \mu\left(e^{\star}\right)^{2}\right) \mathbf{\Upsilon}+\sigma \mu\left(e^{\star}\right)^{2}(\mathbf{\Upsilon} \cdot \mathbf{k}) \mathbf{k} .
$$

In accordance with Or's equations, we choose to write the angular momentum with respect to the center of curvature $O$.

The basic phase space is $T Q$, which is typically parametrized by rotational and translational coordinates and their conjugate momenta. The body angular velocity is $\mathbf{R}^{-1} \dot{\mathbf{R}}$, where $\mathbf{R}$ is the attitude matrix of the body and $(\mathbf{R}, \dot{\mathbf{R}})$, together with translational coordinates, is a point in $T Q$. The body angular momentum coordinates are then related to the body angular velocity coordinates by constant factors given by the principal moments of inertia. The Lagrangian $\mathcal{L}: T Q \rightarrow \mathbb{R}$ in terms of these variables is given explicitly by

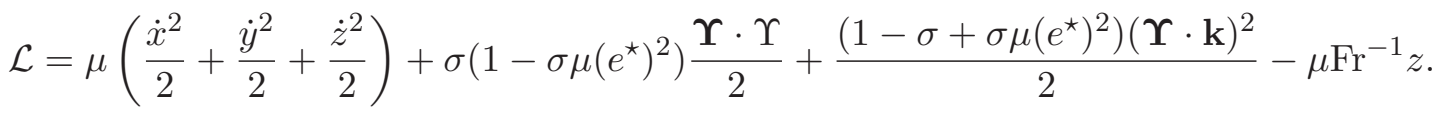

The equations of motion can then be formulated by the Lagrange d'Alembert principle with generalized forces given by the specified sliding friction force. The surface reaction force is a force of constraint that is an intrinsic part of the Euler-Lagrange operator.

\section{Linear theory.}

Equilibria. Equilibria of (3.7) satisfy

$$
\begin{aligned}
\mathbf{\Upsilon} \times \mathbf{k}=0 & \Longrightarrow \mathbf{\Upsilon} \text { and } \mathbf{k} \text { are collinear, } \\
\mathbf{e}_{z} \times \mathbf{k}=0 & \Longrightarrow \mathbf{e}_{z} \text { and } \mathbf{k} \text { are collinear. }
\end{aligned}
$$

Therefore, equilibria satisfy

$$
\dot{x}=\dot{y}=x=y=\Upsilon_{x}=\Upsilon_{y}=l=m=0, \quad \Upsilon_{z}=\text { constant }, \quad n= \pm 1
$$

If we restrict the angular momentum about $\mathbf{q}(\mathrm{cf} .(3.10))$ to a certain value, i.e., $\Upsilon_{Q}=$ $-\left(1+e^{\star}\right) \sigma$, we have two equilibria given by

$$
\dot{x}=\dot{y}=x=y=\Upsilon_{x}=\Upsilon_{y}=l=m=0, \quad \Upsilon_{z}=1, \frac{1+e^{\star}}{1-e^{\star}}, \quad n= \pm 1 .
$$

These equilibria correspond to the noninverted and inverted states of the tippe top shown in Figure 1.1.

Linearization. Linearizing the equations of motion about these states $\left(n=n^{o}= \pm 1\right.$, $\Upsilon_{z}=\Upsilon_{z}^{o}$ ) and writing the resulting equations in terms of the complex variables

$$
V_{C}=\dot{x}+i \dot{y}, \quad \Lambda=\Upsilon_{x}+i \Upsilon_{y}, \quad \Phi=l+i m
$$


we obtain

$$
\begin{aligned}
\dot{V}_{C} & =-\nu A V_{C}-i \nu B \Lambda+i \nu C \Phi, \\
\dot{\Lambda} & =i \nu D V_{C}+(\nu E+i F) \Lambda+(\nu G+i H) \Phi, \\
\dot{\Phi} & =-i n^{o} \sigma \Lambda+i \sigma \Upsilon_{z}^{o} \Phi,
\end{aligned}
$$

where we have introduced the following real parameters to ensure clarity of (4.2):

$$
\begin{gathered}
A=\frac{1}{\mu}, \quad B=\frac{1}{\mu} \sigma\left(e^{\star} n^{o}+1\right), \quad C=\frac{\left(\sigma e^{\star}+n^{o}(\sigma-1)\right) \Upsilon_{z}^{o}}{\mu}, \quad D=\frac{\left(e^{\star} n^{o}+1\right)}{\sigma\left(1-\mu\left(e^{\star}\right)^{2}\right)}, \\
E=\frac{\left(e^{\star} n^{o}+1\right)^{2}}{1-\mu\left(e^{\star}\right)^{2}}, \quad F=\frac{n^{o} \mu\left(e^{\star}\right)^{2} \sigma}{1-\mu\left(e^{\star}\right)^{2}}, \\
G=\frac{\left(e^{\star} n^{o}+1\right)\left(\sigma e^{\star}+n^{o}(\sigma-1)\right) \Upsilon_{z}^{o}}{\sigma\left(1-\mu\left(e^{\star}\right)^{2}\right)}, \quad H=\frac{-\mu\left(e^{\star}\right)^{2} \sigma^{2} \Upsilon_{z}^{o}+\mu e^{\star} \operatorname{Fr}^{-1}}{\sigma\left(1-\mu\left(e^{\star}\right)^{2}\right)} .
\end{gathered}
$$

To obtain Or's linearized model, set $n^{o}=1, \Upsilon_{z}{ }^{o}=1$ in (4.2) and express the equations in terms of the velocity of the center of curvature, $V_{0}$, and the angular velocity, $\boldsymbol{\omega}$, through the following change of dependent variables: $V_{C}=V_{0}+e^{\star} i(\Phi-\boldsymbol{\omega})$, and $\sigma \Lambda=\boldsymbol{\omega}+(\sigma-1) \Phi[11$, eq. (11), p. 602].

If we assume a solution of the form $e^{\lambda t} \phi$, we obtain the following eigenvalue problem:

$$
\lambda \phi=\nu \mathbf{C} \phi+i \mathbf{K} \phi
$$

where

$$
\mathbf{C}=\left[\begin{array}{ccc}
-A & -i B & i C \\
i D & E & G \\
0 & 0 & 0
\end{array}\right], \quad \mathbf{K}=\left[\begin{array}{ccc}
0 & 0 & 0 \\
0 & i F & i H \\
0 & -n^{o} \sigma & \Upsilon_{z}^{o} \sigma
\end{array}\right]
$$

Numerical stability analysis. Here we briefly confirm Or's numerical stability results and discuss the stability of the inverted state: $n^{o}=-1, \Upsilon_{z}{ }^{o}=\frac{1+e^{\star}}{1-e^{\star}}$. A numerical linear stability analysis of the governing equation reveals the effect of the Froude number $\mathrm{Fr}^{-1}$, the inertia ratio $\sigma$, and the friction factor $\nu$ as a function of the eccentricity $e^{\star}$. Figure 4.1 shows the growth factor of the eigenvalue with largest real part. When the center of mass is below the center of curvature, we see that a high Froude number destabilizes the noninverted state. A low Froude number implies that gravitational effects are more important than rotational effects, and therefore if the center of mass is below the center of curvature, a low Froude number stabilizes the standing spinning solution. When the center of mass moves above the center of curvature, we see that the Froude number has the opposite effect.

Figure 4.3 shows that the inertia ratio $\sigma$ destabilizes the standing spinning solution for $\sigma<1.14$ when the center of mass is below the center of curvature. As Cohen notes, the inertia ratio of most commercial tippe tops is less than 1, i.e., $\sigma<1$.

Figure 4.5 shows that the inviscid problem is neutrally stable, as expected. As $\nu$ increases, the magnitude of the real part of the eigenvalue initially becomes larger until the friction 


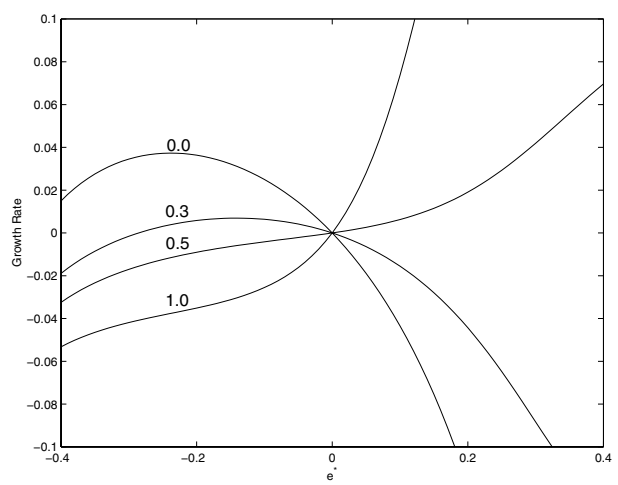

Figure 4.1. The growth factor of the eigenvalue of (4.3) with largest real part is plotted here for varying Froude numbers $\left(\mathrm{Fr}^{-1}=0,0.3,0.5,1.0\right)$ as functions of $e^{\star}$ for the noninverted equilibrium state $n^{\circ}=1$, $\Upsilon_{z}^{o}=1$

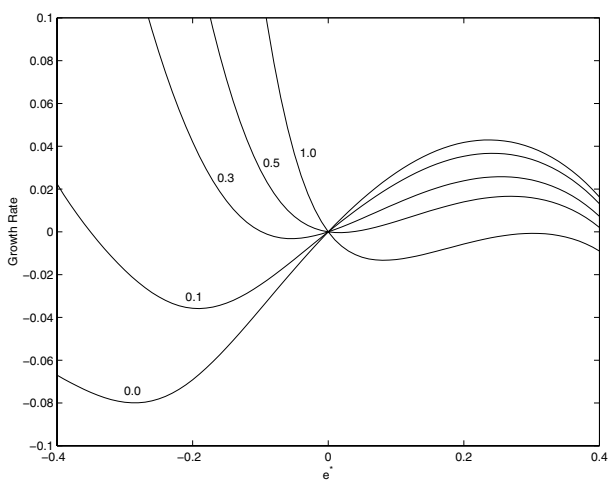

Figure 4.2. The growth factor of the eigenvalue of (4.3) with largest real part is plotted here for varying Froude numbers $\left(\mathrm{Fr}^{-1}=0,0.3,0.5,1.0\right)$ as functions of $e^{\star}$ for the inverted equilibrium state $n^{\circ}=-1$, $\Upsilon_{z}^{o}=\frac{1+e^{\star}}{1-e^{\star}}$.

becomes so large that the ball ceases to slip. The real part of the eigenvalue then begins to decay as the ball tends to roll without slip. In the limit to pure rolling, we see that the nonholonomic problem is also neutrally stable. For $\nu>0$, we also observe that the real part of the eigenvalue is positive (or negative) when the center of gravity of the ball is below (or above) the geometric center. This explains why the inverted state is linearly stable. All of these findings agree with those of Or [11].

Figures 4.2, 4.4, and 4.6 show that the behavior of the inverted state when $e^{\star}<0$ is qualitatively similar to the behavior of the noninverted state with $e^{\star}>0$; however, they are not quantitatively the same. In all of these figures, we see ranges of parameter values where the inverted state is asymptotically stable and unstable when $e^{\star}<0$. We will show later that for parameter values where the inverted state is asymptotically stable and the noninverted state is asymptotically unstable, a heteroclinic orbit exists, and the inverted state is globally asymptotically stable. 


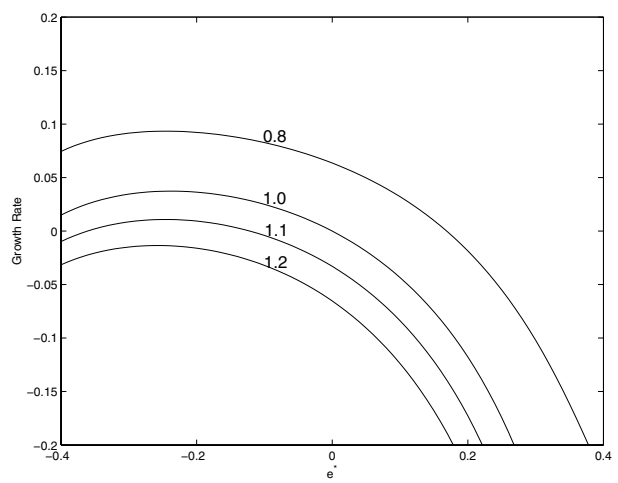

Figure 4.3. The growth factor of the eigenvalue of (4.3) with largest real part is plotted here for varying inertia ratios $(\sigma=0.8,1.0,1.1,1.2)$ as functions of $e^{\star}$ for the noninverted equilibrium state $n^{\circ}=1, \Upsilon_{z}{ }^{\circ}=1$.

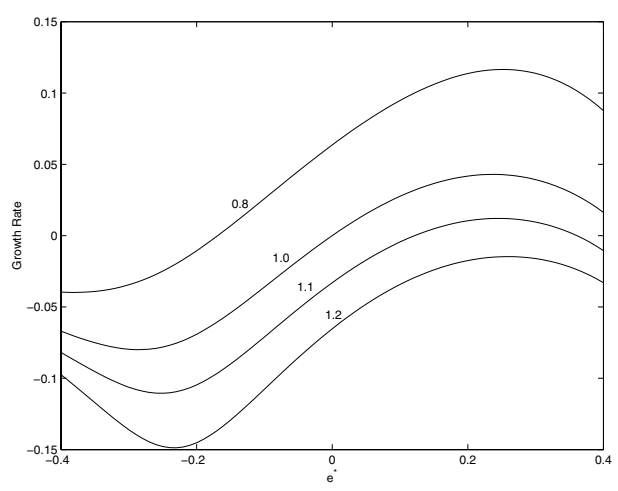

Figure 4.4. The growth factor of the eigenvalue of (4.3) with largest real part is plotted here for varying inertia ratios $(\sigma=0.8,1.0,1.1,1.2)$ as functions of $e^{\star}$ for the inverted equilibrium state $n^{o}=-1, \Upsilon_{z}^{o}=\frac{1+e^{\star}}{1-e^{\star}}$.

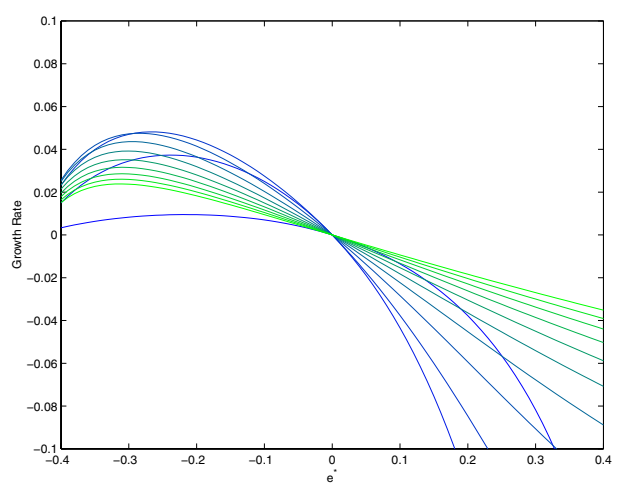

Figure 4.5. The growth factor of the eigenvalue of (4.3) with largest real part is plotted here for $\mathrm{Fr}^{-1}=0$ as functions of $e^{\star}$ for $\nu$ varying from 0.1 (blue) to 5.0 (green) for the noninverted equilibrium state $n^{\circ}=1$, $\Upsilon_{z}{ }^{o}=1$. 


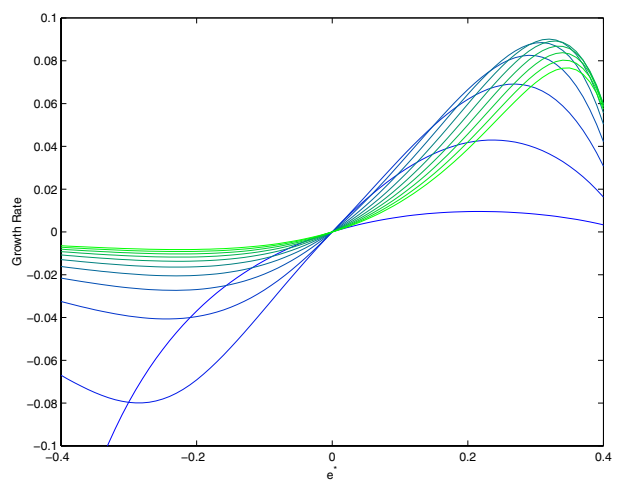

Figure 4.6. The growth factor of the eigenvalue of (4.3) with largest real part is plotted here for $\mathrm{Fr}^{-1}=0$ as functions of $e^{\star}$ for $\nu$ varying from 0.1 (blue) to 5.0 (green) for the inverted equilibrium state $n^{o}=-1$, $\Upsilon_{z}^{o}=\frac{1+e^{\star}}{1-e^{\star}}$.

5. Tippe top modified Maxwell-Bloch equations. If we ignore translational effects, the linearized equations (4.2) simplify:

$$
\begin{aligned}
\dot{\Lambda} & =(\nu E+i F) \Lambda+(\nu G+i H) \Phi, \\
\dot{\Phi} & =-i n^{o} \sigma \Lambda+i \sigma \Upsilon_{z}^{o} \Phi .
\end{aligned}
$$

To obtain these equations more directly, we can ignore translational effects from the outset. The derivation based on this assumption is closely related to deriving the equations of motion for the standard top in a gravitational field. There is ample evidence supporting this assumption:

1. The assumption is rigorously true for small friction. Equation (4.2) shows that when the friction is zero the velocity of the center of mass is fixed. Now consider a perturbation of an inviscid, initially restating state in terms of the dissipation $\nu$. To first order in $\nu$, it is sufficient to use only the zeroth-order velocity of the center of mass, which is by assumption zero. Thus, to first order the center of mass can be assumed to remain fixed. This reasoning can be borne out by application of rigorous perturbation theory. We do not attempt such an analysis in this work.

2. Numerical evidence shows that application of the perturbation theory of eigenvalues with or without the effect of translation produces the same stability results.

3. We will show that the position of the center of mass remains fixed for all extrema of the constrained energy (see section 6). Thus, translational effects are negligible in the steady-state behavior of trajectories in the neighborhood of equilibria (critical points of the constrained energy).

4. Simulation shows that the translational energy of the tippe top is negligible (see Figure 7.2) and that the tippe top rotates much more than it translates.

Equation (5.1) can be rewritten in terms of $\Phi$ alone:

$$
\ddot{\Phi}+i a \dot{\Phi}+b \dot{\Phi}+i c \Phi+d \Phi=0
$$


where

$$
\begin{aligned}
& a=\frac{\sigma\left(\Upsilon_{z}^{o}-\left(\Upsilon_{z}^{o}-n^{o}\right)\left(e^{\star}\right)^{2} \mu\right)}{-1+\left(e^{\star}\right)^{2} \mu}, \\
& b=-\frac{\left(1+n^{o} e^{\star}\right)^{2} \nu}{-1+\left(e^{\star}\right)^{2} \mu}, \\
& c=\frac{\nu \Upsilon_{z}^{o}\left(1+n^{o} e^{\star}\right)\left(-\left(n^{o}\right)^{2}(-1+\sigma)+\sigma\right)}{-1+\left(e^{\star}\right)^{2} \mu}, \\
& d=\frac{\operatorname{Fr}^{-1} e^{\star} \mu n^{o}}{-1+\left(e^{\star}\right)^{2} \mu} .
\end{aligned}
$$

Notice that (5.2) is in the form of the modified Maxwell-Bloch equations (2.1).

Now let us check the stability criteria for modified Maxwell-Bloch systems given in (2.2). The stability criteria for the noninverted state $\left(n^{o}=1, \Upsilon_{z}^{o}=1\right)$ are

$$
\begin{aligned}
1-\mu\left(e^{\star}\right)^{2} & >0, \\
\left(1+e^{\star}\right) \nu\left(\operatorname{Fr}^{-1} e^{\star}\left(1+e^{\star}\right) \mu\left(-1+\mu\left(e^{\star}\right)^{2}\right)+\left(1+e^{\star}\right)^{5} \nu^{2}+\sigma\left(-1+\sigma+e^{\star}\left(e^{\star} \mu+\sigma\right)\right)\right) & >0, \\
-\left(1+e^{\star}\right)^{2} \nu^{2}\left(1+\operatorname{Fr}^{-1} e^{\star}\left(1+e^{\star}\right)^{2} \mu-\sigma-e^{\star}\left(e^{\star} \mu+\sigma\right)\right) & >0 .
\end{aligned}
$$

Likewise, the stability criteria for the inverted state $\left(n^{o}=-1, \Upsilon_{z}^{o}=\frac{1+e^{\star}}{1-e^{\star}}\right)$ are

$$
\begin{aligned}
& 1-\mu\left(e^{\star}\right)^{2}>0, \\
& \nu \frac{\left(-\operatorname{Fr}^{-1}\left(-1+e^{\star}\right)^{3} e^{\star} \mu\left(-1+\mu\left(e^{\star}\right)^{2}\right)+\left(-1+e^{\star}\right)^{7} \nu^{2}\right)}{\left(1-e^{\star}\right)\left(-1+\mu\left(e^{\star}\right)^{2}\right)} \\
& +\nu \frac{\left(-1-e^{\star}+2\left(e^{\star}\right)^{2} \mu\right) \sigma\left(-1-e^{\star}+\sigma+\left(e^{\star}\right)^{2}(\mu-\sigma-2 \mu \sigma)+\left(e^{\star}\right)^{3}(\mu+2 \mu \sigma)\right)}{\left(1-e^{\star}\right)\left(-1+\mu\left(e^{\star}\right)^{2}\right)}>0, \\
& \nu^{2}\left(-1+\sigma+e^{\star}\left(-2+\operatorname{Fr}^{-1} \mu+\sigma\right)\right) \\
& +\nu^{2}\left(\left(e^{\star}\right)^{2}\left(-1+\left(1+e^{\star}\right)^{2}+\operatorname{Fr}^{-1}\left(-2+e^{\star}\right)\left(2+\left(-2+e^{\star}\right) e^{\star}\right)\right) \mu-\sigma-e^{\star} \sigma\right)>0 .
\end{aligned}
$$

For the parameter values explored, the first two inequalities are always satisfied. The last inequality is satisfied precisely in the regions where the growth rate of the eigenvalue of (4.3) with largest real part is negative. We observe that this inequality is independent of the friction factor unless $\nu=0$. Thus, we conclude that stability is independent of the magnitude of $\nu$. Figures 5.1 and 5.2 superpose plots of the growth rate with the value of the last inequality in (5.3).

Can we reduce (5.2) any further? The answer is no because of the remarks made in section 2. In particular, it can be shown that, without the complex and usual damping terms, i.e., $b=0$ and $c=0$ or $\nu=0$, the gravitationally stable noninverted state cannot become asymptotically unstable. Moreover, the gyroscopically stabilized state can be asymptotically stable if and only if the complex and usual damping terms are present and in the right ratio. In summary, the normal form for the tippe top in the neighborhood of inversion (a dissipationinduced instability) is described by the modified Maxwell-Bloch equations. 


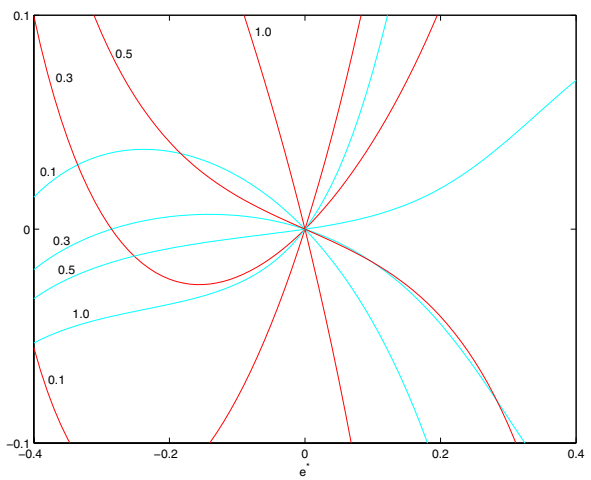

Figure 5.1. The growth factor of the eigenvalue of (4.3) with largest real part (cyan curves) and the third inequality in the stability criteria given in (5.3) (red curves) are plotted here for varying Froude numbers $\left(\mathrm{Fr}^{-1}=0,0.3,0.5,1.0\right)$ as functions of $e^{\star}$. Here we consider the noninverted state $n^{o}=1, \Upsilon_{z}^{o}=1$.

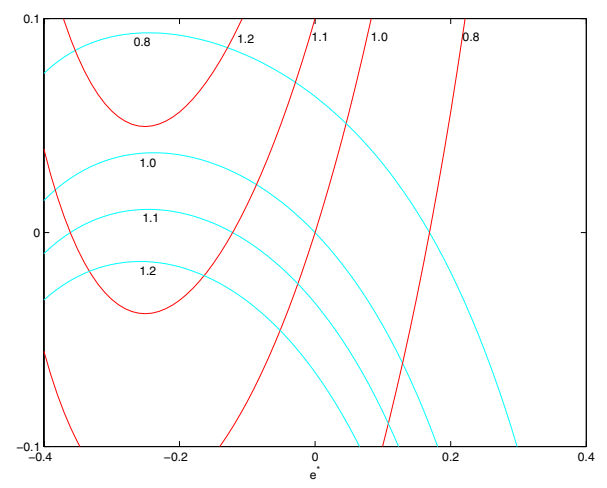

Figure 5.2. The growth factor of the eigenvalue of (4.3) with largest real part (cyan curves) and the third inequality in the stability criteria given in (5.3) (red curves) are plotted here for varying inertia ratios $(\sigma=0.8,1.0,1.1,1.2)$ as functions of $e^{\star}$. Here we consider the noninverted state $n^{o}=1, \Upsilon_{z}^{o}=1$.

\section{Heteroclinic connection.}

Tippe top asymptotic states. To establish the existence of a heteroclinic orbit connecting the asymptotically stable and unstable states of the theoretical tippe top, we will invoke LaSalle's invariance principle. Consider a vector field $\chi$ on a manifold $P$. Let $V$ be a Lyapunov function with negative semidefinite orbital derivative: $V_{t} \leq 0$ for all $z \in P$. We define the set $\aleph:=\left\{z \in P \mid V_{t}(z)=0\right\}$.

Theorem 6.1 (LaSalle's principle). Let $z:[0, \infty) \rightarrow P$ be an integral curve of a vector field $\chi$ with initial condition $z(0)=z_{0}$. Suppose there is a positively invariant set (trapping region) $M$ such that $z(t) \in M$ for all $t \geq 0$. Then $z(t)$ converges to the largest subset of $\aleph \cap M$ that is invariant under the flow of $\chi$ for all $t$, positive and negative.

We will now apply this principle to (3.7), the governing equation of the viscous ball. We write the energy of the viscous ball as

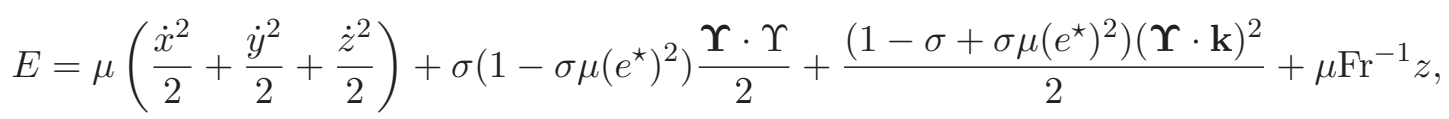


which is a sum of translational, rotational, and gravitational effects. The energy's orbital derivative is given by

$$
E_{t}=-\nu\left\|\mathbf{v}_{Q}\right\|^{2}
$$

where $\left\|\mathbf{v}_{Q}\right\|$ is the $\ell_{2}$-norm of the dimensionless slip velocity. The energy's orbital derivative implies that the energy decreases monotonically until the slip velocity vanishes.

No-slip no-force problem. We now mention some properties of the asymptotic state, where the slip velocity vanishes and energy is conserved. The governing equations (3.7) without slip reduce to the system

$$
\begin{aligned}
\ddot{x} & =0 \\
\ddot{y} & =0, \\
\dot{\boldsymbol{\Upsilon}} & =\frac{1}{1-\sigma \mu\left(e^{\star}\right)^{2}}\left(\sigma \mu\left(e^{\star}\right)^{2}(\mathbf{\Upsilon} \cdot \mathbf{k})(\mathbf{k} \times \mathbf{\Upsilon})+e^{\star} f_{z}\left(\mathbf{e}_{z} \times \mathbf{k}\right)\right), \\
\dot{\mathbf{k}} & =\sigma \mathbf{\Upsilon} \times \mathbf{k},
\end{aligned}
$$

where there is no tangential surface force. Clearly, this system is Hamiltonian, i.e., it conserves energy. It is readily shown that

$$
\dot{\mathbf{v}_{Q}}=0 \Longrightarrow \dot{n}=0 \Longrightarrow \dot{\Upsilon}_{z}=0 \Longrightarrow \Upsilon \cdot\left(\mathbf{e}_{z} \times \mathbf{k}\right)=0 \Longrightarrow \dot{x}=\dot{y}=0 .
$$

Solutions are therefore defined by level sets of $l^{2}+m^{2}$ and $\left(\Upsilon_{x}\right)^{2}+\left(\Upsilon_{y}\right)^{2}$ [6]. For $n \neq \pm 1$ these solutions are described by precession, spin about the axis of symmetry, and no nutation. These are Ebenfeld's tumbling solutions [6].

The abundance of invariant quantities in the no-slip, no-force problem severely limits the behavior of solutions to (6.3). In fact, the analysis above shows that the angular momentum about the vertical and the nutation angle fully determine solutions of (6.3). The nutation angle $\theta$ is the angle which the axis of symmetry makes with the vertical $\mathbf{e}_{z}$ :

$$
n=\cos (\theta) .
$$

We reasonably suppose that the initially sliding, viscous tippe top will tend to an asymptotic state, where $\Upsilon_{z}$ and $n$ extremize the energy subject to the constraints in the problem. Thus, we suppose that $\aleph$ is the set of all energy-conserving asymptotic solutions defined by $\Upsilon_{z}$ and $n$ which extremize the constrained energy.

6.1. Energy-momentum minimization. We now determine all minima of the total energy subject to the angular momentum constraint, $\Upsilon_{Q}=-\sigma\left(1+e^{\star}\right)$ (cf. (3.10)), and the attitude constraint, $\mathbf{k} \cdot \mathbf{k}=1$. In other words, we find the extrema of the augmented energy $h=$ $E+\lambda \Upsilon_{Q}+\hat{\lambda} \mathbf{k} \cdot \mathbf{k}$, where $\lambda$ and $\hat{\lambda}$ are Lagrange multipliers. Extrema of $h$ satisfy

$$
\dot{x}=\dot{y}=\dot{z}=0,
$$

which indicates that the center of mass and nutation angle remain fixed. The angular momentum in terms of $n$ takes the form

$$
\mathbf{\Upsilon}=\frac{\lambda}{\sigma^{2}}\left(\left((\sigma-1) n+e^{\star} \sigma\right) \mathbf{k}+\mathbf{e}_{z}\right),
$$


where

$$
\lambda=\frac{\left(1+e^{\star}\right) \sigma^{2}}{\left(-1+n^{2}\right)\left(-1+\mu\left(e^{\star}\right)^{2}\right)+\left(n+e^{\star}\right)^{2} \sigma} .
$$

Once the angular momentum takes the above form, the slip velocity vanishes: $\mathbf{v}_{Q}=0$. This result is expected since extrema of the energy should have the same properties as the no-slip, no-force problem. With an angular momentum of this form, extrema of the energy satisfy

$$
\begin{aligned}
& \frac{\lambda^{2}}{\sigma^{2}}\left[\left(\mu\left(e^{\star}\right)^{2}-1\right)\left((\sigma-1) n+e^{\star} \sigma\right)^{2}\right] \mathbf{k}+2 \hat{\lambda} \mathbf{k} \\
& +\frac{\lambda^{2}}{\sigma^{2}}\left[(1-\sigma) n-e^{\star} \sigma-\mu\left(e^{\star}\right)^{2} n\right] \mathbf{e}_{z}+\mu e^{\star} \operatorname{Fr}^{-1} \mathbf{e}_{z}=0 .
\end{aligned}
$$

When $\mathbf{e}_{z}$ and $\mathbf{k}$ are linearly dependent, we obtain the solutions $n= \pm 1$ and $\mathbf{\Upsilon}=\Upsilon_{z} \mathbf{e}_{z}$ with $\Upsilon_{z}=1, \frac{1+e^{\star}}{1-e^{\star}}$, which correspond to the inverted and noninverted states, respectively. When the two vectors are linearly independent, we can choose $\hat{\lambda}$ so that the coefficient of $\mathbf{k}$ vanishes. The coefficient of $\mathbf{e}_{z}$ vanishes when the following polynomial equation in $n$ is satisfied:

$$
\left[(1-\sigma) n-e^{\star} \sigma-\mu\left(e^{\star}\right)^{2} n+\frac{\sigma^{2}}{\lambda^{2}} \mu e^{\star} \mathrm{Fr}^{-1}\right]=0 .
$$

This reduction to a one-dimensional problem in terms of components of the energy that are independent of the friction factor $\nu$, and in terms of quantities in the plane defined by the vertical and the axis of symmetry, agrees with Ebenfeld's result [6]. This equation can be rewritten as a quartic polynomial of the form

$$
a_{0} n^{4}+a_{1} n^{3}+a_{2} n^{2}+a_{3} n+a_{4}=0
$$

where the coefficients are functions of the parameters $\mu, \mathrm{Fr}^{-1}, \sigma$, and $e^{\star}$.

Heteroclinic connection existence criteria. We are not interested in explicitly solving the quartic equation (6.4). Rather, we are interested in knowing if all of the roots of this equation are outside the unit disc in the complex plane, since if $|n|>1$, the extrema violates the constraint $\mathbf{k} \cdot \mathbf{k}=1$. We can determine whether all roots satisfy this criterion by the following transformation in the complex domain:

$$
n=\frac{-1+z}{1+z}
$$

which describes a mapping from the region outside the open disc $|n|>1$ to the open left half-plane in $z$. In terms of $z$ the quartic polynomial becomes

$$
r_{0} z^{4}+r_{1} z^{3}+r_{2} z^{2}+r_{3} z+r_{4}=0 .
$$

Specific values of these coefficients are provided in the appendix.

We now invoke the Liénard-Chipart criterion for the stability of quartic polynomials [7, p. 221]. 
Theorem 6.2 (Liénard-Chipart criterion). Necessary and sufficient conditions for all the roots of $r_{0} z^{4}+r_{1} z^{3}+r_{2} z^{2}+r_{3} z+a_{4}=0$ to have negative real parts can be given by

$$
r_{0}>0, \quad r_{1}>0, \quad r_{2}>0, \quad r_{3}>0, \quad r_{4}>0, \quad d=r_{1} r_{2} r_{3}-r_{0} r_{3}^{2}-r_{4} r_{1}^{2}>0 .
$$

In Figures 6.1, 6.2, and 6.3, we show the values of the coefficients and $d$ for the cases $\mathrm{Fr}^{-1}=0.3,0.2,0.1, \sigma=1, \mu=2.28$, and variable $e^{\star}$. Comparing these figures with the linear-stability plot for the noninverted and inverted equilibria in Figure 7.3, we observe that there are no other extrema in which the noninverted state is asymptotically unstable and the inverted state is asymptotically stable. In particular, for $\mathrm{Fr}^{-1}=0.3,0.2,0.1$ and $e^{\star}<0$, we observe that there are no other extrema in these ranges, respectively: $-0.1<e^{\star}<0.0$, $-0.2<e^{\star}<0.0$, and $-0.35<e^{\star}<0$. By evaluating the energy at the noninverted and inverted states, it can be shown that in these parameter ranges the inverted and noninverted states are absolute minima and maxima of the constrained energy, respectively.

In this regime of parameter values, the inverted state is the only point that remains in $\aleph$. By the theorems of Barbashin and Krasovskii, the inverted state is globally asymptotically stable in the parameter ranges, where the only extrema are the inverted and noninverted

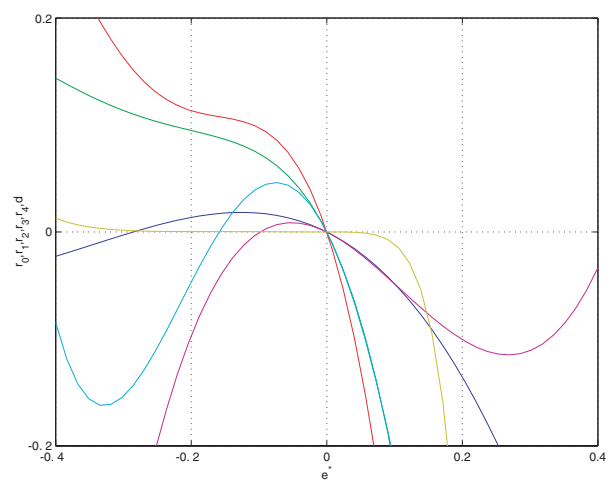

Figure 6.1. Here we plot the coefficients of the quartic in $z=\frac{n+1}{1-n}$ for $\mu=2.28, \mathrm{Fr}^{-1}=0.3, \sigma=1.0$, and variable $e^{\star}$.

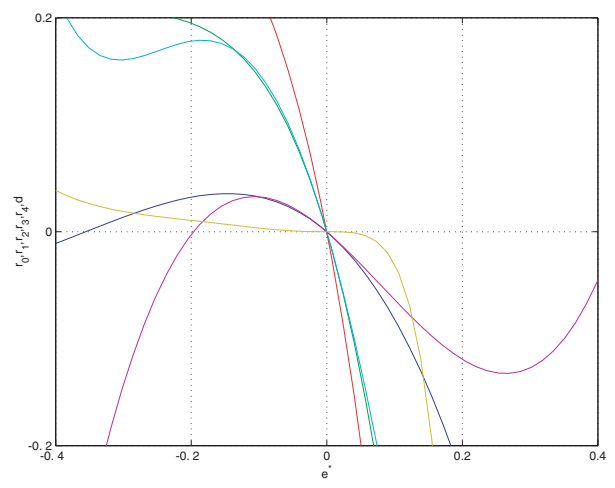

Figure 6.2. Here we plot the coefficients of the quartic in $z=\frac{n+1}{1-n}$ for $\mu=2.28, \mathrm{Fr}^{-1}=0.2, \sigma=1.0$, and variable $e^{\star}$. 


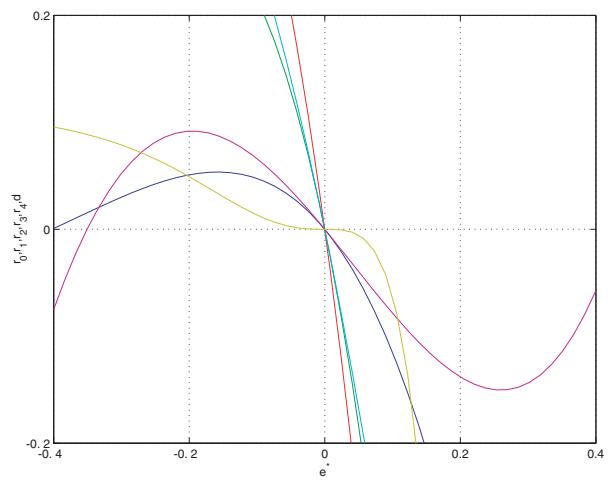

Figure 6.3. Here we plot the coefficients of the quartic in $z=\frac{n+1}{1-n}$ for $\mu=2.28, \mathrm{Fr}^{-1}=0.1, \sigma=1.0$, and variable $e^{\star}$.

states [8]. When the extrema include the tumbling solution, where $1-n^{2} \neq 0$, the heteroclinic connection does not exist. Ebenfeld provides specific criteria for the Lyapunov stability of this limit cycle. By the properties of the asymptotic states of the no-slip, no-force problem, it is clear this limit cycle should be neutrally stable.

The correspondence between the linear and nonlinear results is much more profound. We observe that the Hurwitz coefficients, which dictate stability of the polynomial in $z$, and hence determine the allowable nutation angles of extrema, are always $r_{0}$ and $r_{4}$. The appendix provides explicit expressions for these terms. Comparing the expressions for $r_{0}$ and $r_{4}$ with the stability criteria for the tippe top modified Maxwell-Bloch system equations (5.3) and (5.4), we observe that $r_{0}$ and $r_{4}$ are constant multiples of the third inequalities in (5.3) and (5.4). In section 5 , we showed that these inequalities determine the linear stability of the noninverted and inverted states of the tippe top. Thus, because of dissipation the linear analysis supplies all of the information needed to determine existence of the heteroclinic connection.

7. Simulation. We first reproduce some results in the literature by numerical integration of the governing equations (3.7). We time-integrate the equations of motion using the adaptive Runge-Kutta method. Plots of the nutation angle, precessional velocity, and spin velocity of the ball from Cohen's pioneering work are shown in Figure 7.1. The spin velocity figure illustrates conservation of angular momentum as the spin velocity changes direction when the axis of symmetry becomes horizontal. Figure 7.2 shows plots of nutation angle, spin velocity, and total energy as functions of time from Or's seminal work. The plot of the total, rotational, translational, and potential energies revealed that rotational, gravitational, and dissipative effects dominate translational effects.

We will now discuss data from simulations which confirm the local and global stability analysis in this paper. We consider tippe top inversion and tumbling for three cases: $\mathrm{Fr}^{-1}=0.1$ (high Froude number), $\mathrm{Fr}^{-1}=0.2$ (moderate Froude number), and $\mathrm{Fr}^{-1}=0.3$ (low Froude number). Other parameters are valued at $\mu=2.28, \sigma=1.0$, and $\nu=0.5$. Initial conditions for inversion are $\Upsilon_{x}=\Upsilon_{y}=0.0, \Upsilon_{z}=1.0, l=m=0.0676, n=0.9954, x=y=$ $-0.0101, \dot{x}=0.1010$, and $\dot{y}=0.0309$. Initial conditions for tumbling are $\Upsilon_{x}=\Upsilon_{y}=0.0$, $\Upsilon_{z}=\left(1.0+e^{\star}\right) /\left(1.0-e^{\star}\right), 1.0, l=m=0.0676, n=\mp 0.9954, x=y=-0.0101, \dot{x}=0.1010$, and $\dot{y}=0.0309$. 

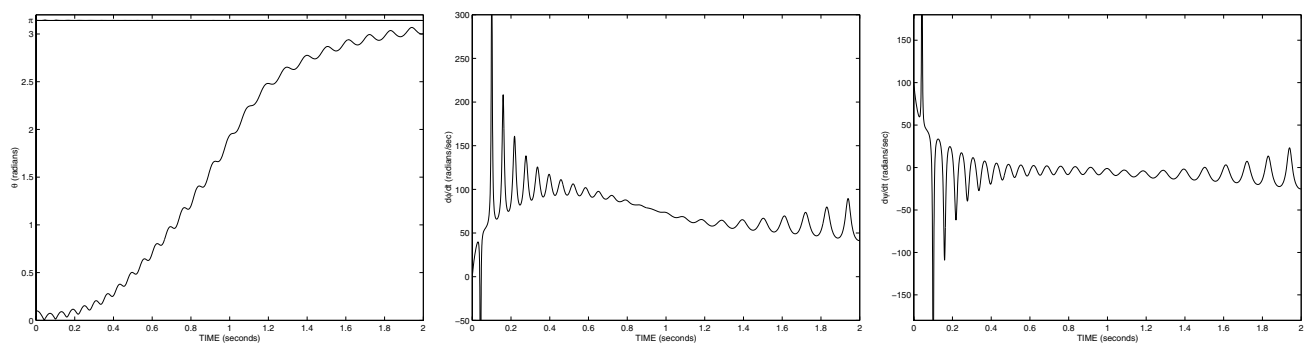

Figure 7.1. From left: we show the nutation angle, the precessional velocity, and the spin rate as functions of time from Cohen's pioneering work [5].
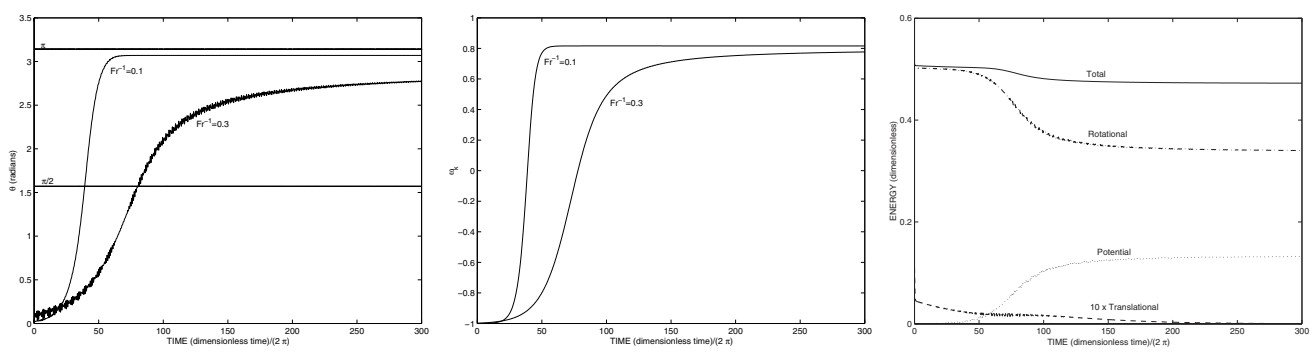

Figure 7.2. From left: we show the nutation angle, the spin velocity, and the total energy and its components as functions of time from Or's seminal work [11].

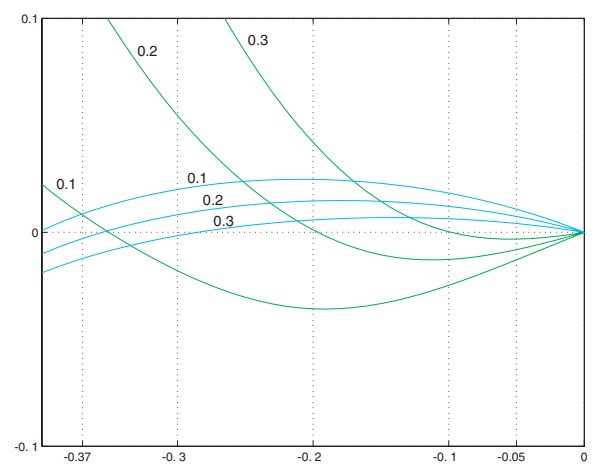

Figure 7.3. Here we plot the growth factor of the eigenvalue of (4.3) with largest real part for both equilibria: the cyan curve corresponds to the noninverted state $\left(n^{\circ}=1, \Upsilon_{z}{ }^{o}=1\right)$, and the green curve corresponds to the inverted state $\left(n^{o}=-1, \Upsilon_{z}^{o}=\frac{1+e^{\star}}{1-e^{\star}}\right)$ for varying Froude numbers $\left(\mathrm{Fr}^{-1}=0.1,0.2,0.3\right)$. We use this stability plot to select appropriate parameter values for simulation of inversion and tumbling.

The center-of-mass offset values $e^{\star}$ are chosen so that the noninverted state is asymptotically unstable and the inverted state is asymptotically stable (for inversion) and asymptotically unstable (for tumbling). We determine these values of $e^{\star}$ using the linear-stability plot in Figure 7.3. For low, moderate, and high Froude numbers, we pick $e^{\star}=(-0.05,-0.2)$, $(-0.1,-0.3),(-0.2,-0.37)$, respectively. The first $e^{\star}$ in the ordered pair corresponds to tippe top inversion and the second to tumbling. Figures 7.4, 7.6, and 7.8 show trajectories of the axis of symmetry on the unit ball for tippe top inversion. Figures 7.5, 7.7, and 7.9 show 

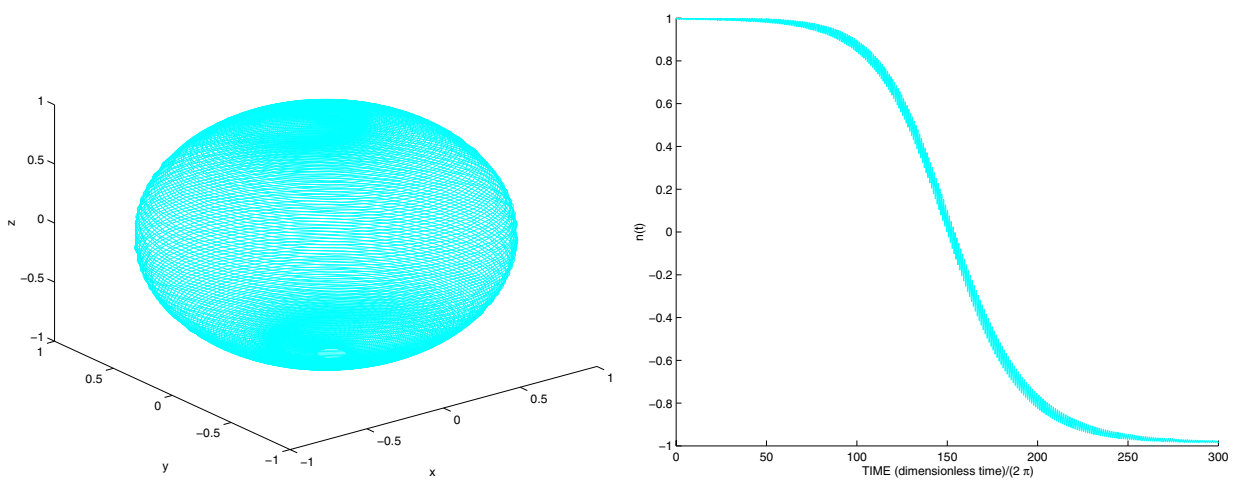

Figure 7.4. Here we show the heteroclinic connection between the asymptotically unstable and stable states of the tippe top for $\mathrm{Fr}^{-1}=0.1$. Specifically, we show the trajectory of the axis of symmetry for a case when $\chi$, the largest invariant set, is the isolated, asymptotically stable equilibrium point. Clicking on the above image displays the associated movie (60135_01.mpg).
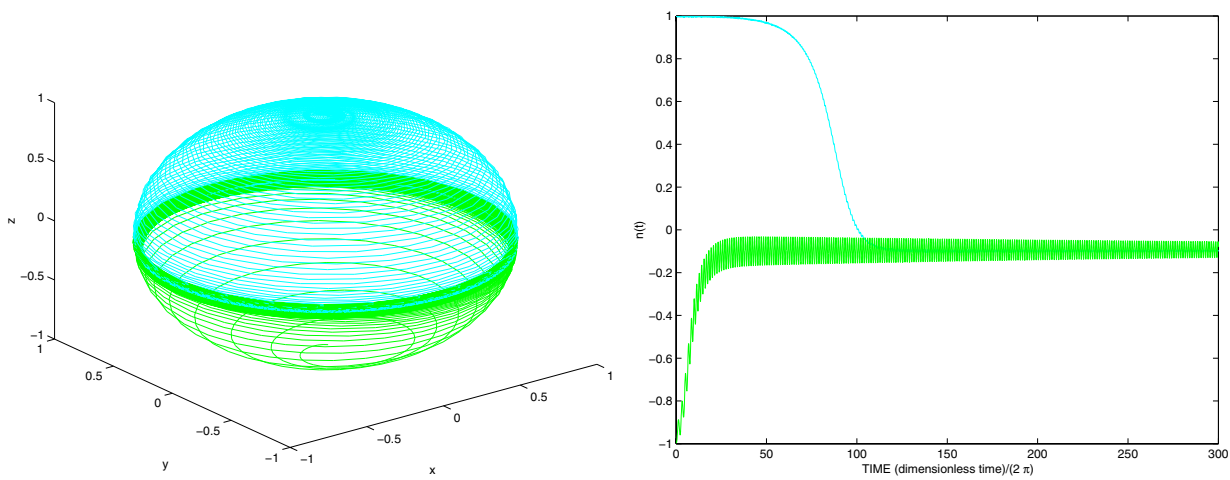

Figure 7.5. This figure shows the trajectory of the axis of symmetry for a case when $\chi$ are only limit cycles defined by curves of constant energy, nutation angle, and angular momentum about the vertical for $\mathrm{Fr}^{-1}=0.1$. The parameter values for this case yield asymptotically unstable inverted and noninverted states. Clicking on the above image displays the associated movie (60135_02.mpg).

trajectories of the axis of symmetry on the unit ball for tippe top tumbling. Like Or, we observe that when the Froude number is small (weak spin relative to gravity) the tippe top takes longer to invert. As the Froude number increases, we observe that the tippe top flips more rapidly.

Six animations that correspond to each case above and that illustrate the phenomenon of tippe top inversion and tumbling can be found at http://www.acm.caltech.edu/ nawaf/ tippetop.html. The animations show the evolution of the axis of symmetry and center of mass. It is very clear from these animations that the tippe top rotates much more than it translates: numerical evidence that translation of the mass center is not necessary to understand the linearized behavior of the ball. 

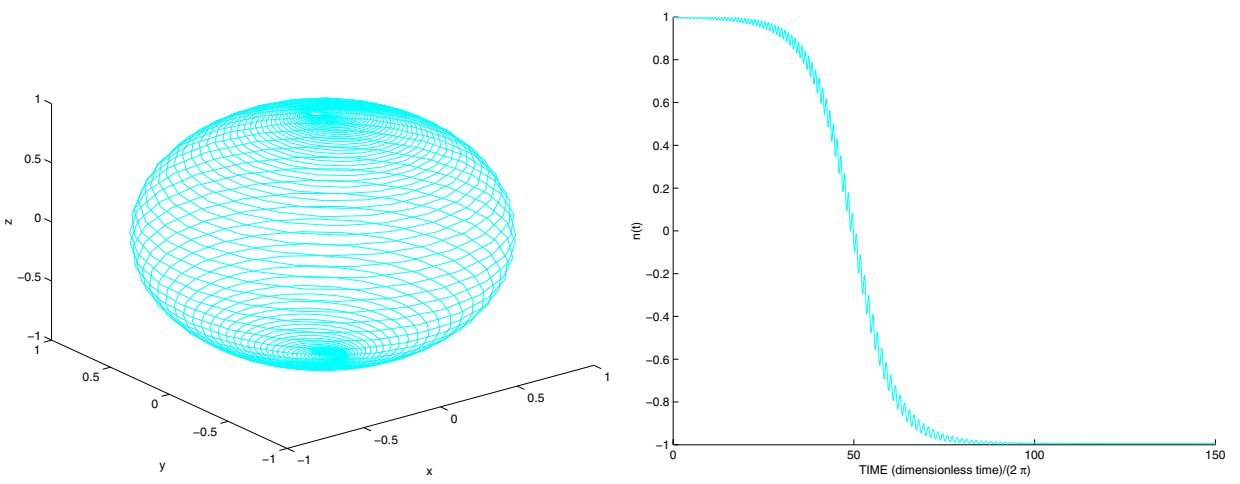

Figure 7.6. Here we show the heteroclinic connection between the asymptotically unstable and stable states of the tippe top for $\mathrm{Fr}^{-1}=0.2$. Specifically, we show the trajectory of the axis of symmetry for a case when $\chi$, the largest invariant set, is the isolated, asymptotically stable equilibrium point. Clicking on the above image displays the associated movie (60135_03.mpg).
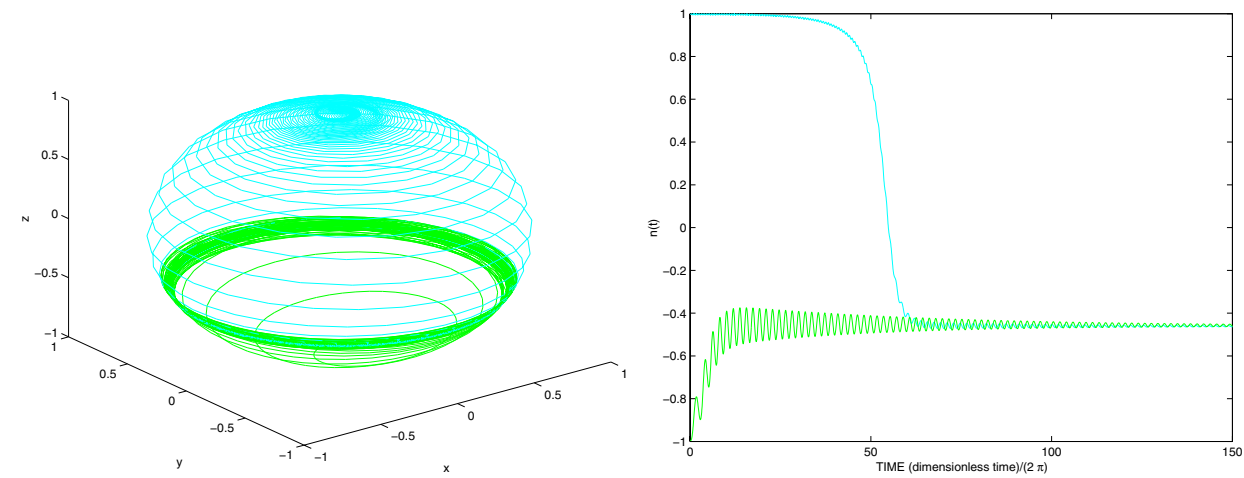

Figure 7.7. This figure shows the trajectory of the axis of symmetry for a case when $\chi$ are only limit cycles defined by curves of constant energy, nutation angle, and angular momentum about the vertical for $\mathrm{Fr}^{-1}=0.2$. The parameter values for this case yield asymptotically unstable inverted and noninverted states. Clicking on the above image displays the associated movie (60135_04.mpg).
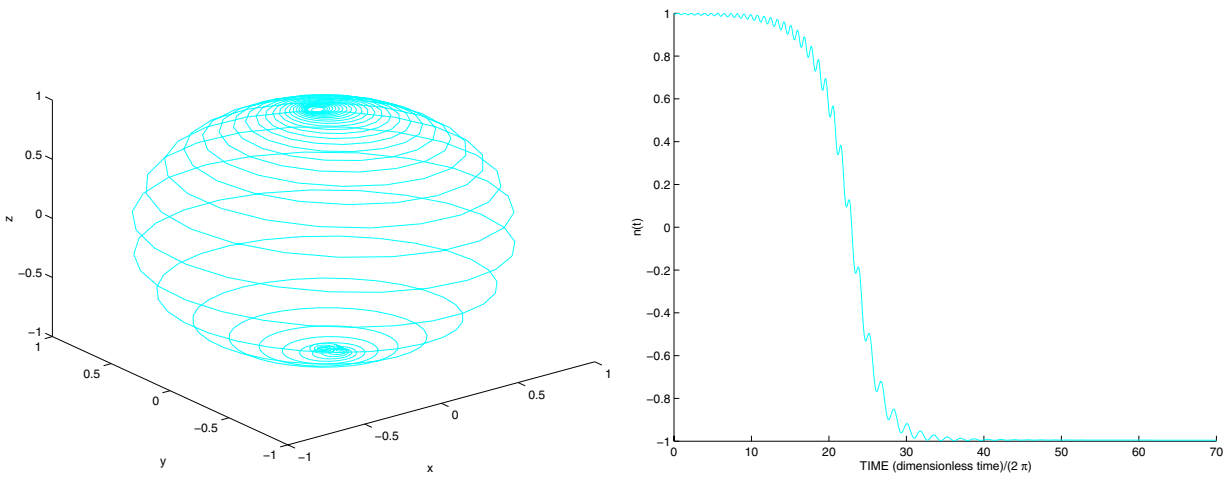

Figure 7.8. Here we show the heteroclinic connection between the asymptotically unstable and stable states of the tippe top for $\mathrm{Fr}^{-1}=0.3$. Specifically, we show the trajectory of the axis of symmetry for a case when $\chi$, the largest invariant set, is the isolated, asymptotically stable equilibrium point. Clicking on the above image displays the associated movie (60135_05.mpg). 

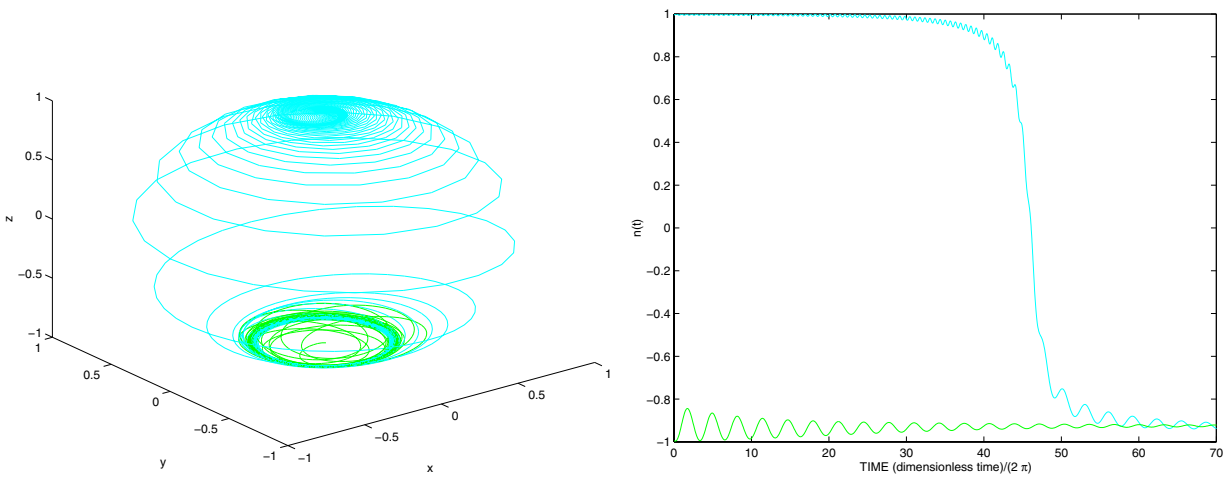

Figure 7.9. This figure shows the trajectory of the axis of symmetry for a case when $\chi$ are only limit cycles defined by curves of constant energy, nutation angle, and angular momentum about the vertical for $\mathrm{Fr}^{-1}=0.3$. The parameter values for this case yield asymptotically unstable inverted and noninverted states. Clicking on the above image displays the associated movie (60135_06.mpg).

8. Future directions. Possible future directions include investigating other axisymmetric physical systems described by the modified Maxwell-Bloch equations (e.g., rotating beam and levitron). For a discussion of the related, but different, rising egg, please see Bou-Rabee, Marsden, and Romero [3].

\section{Appendix.}

Normal reaction force. An explicit expression for the surface normal reaction force follows:

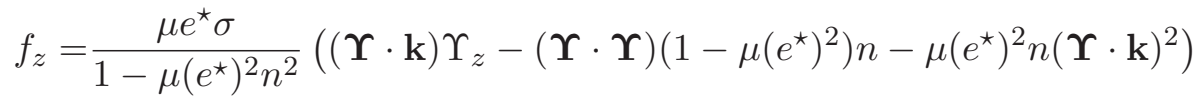

$$
\begin{aligned}
& +\frac{\mu e^{\star}}{1-\mu\left(e^{\star}\right)^{2} n^{2}}\left(1+e^{\star} n\right)\left(\mathbf{k} \cdot \mathbf{f}_{f}\right)+\frac{\mu\left(1-\mu\left(e^{\star}\right)^{2}\right)}{\sigma\left(1-\mu\left(e^{\star}\right)^{2} n^{2}\right)} \operatorname{Fr}^{-1} \text {. }
\end{aligned}
$$

Governing equations in component form. The attitude equations are

$$
\begin{aligned}
\dot{l} & =\left(-\Upsilon_{z} m+\Upsilon_{y} n\right) \sigma, \\
\dot{m} & =\left(\Upsilon_{z} l-\Upsilon_{x} n\right) \sigma, \\
\dot{n} & =\left(-\Upsilon_{y} l+\Upsilon_{x} m\right) \sigma .
\end{aligned}
$$

The translational equations are

$$
\begin{aligned}
& \ddot{x}=-\frac{\nu}{\mu}\left(\dot{x}-e^{\star} \dot{l}+(\sigma-1)(\mathbf{\Upsilon} \cdot \mathbf{k})-\sigma \Upsilon_{y}\right)=\frac{\sigma}{\mu} f_{x}, \\
& \ddot{y}=-\frac{\nu}{\mu}\left(\dot{y}-e^{\star} \dot{m}-(\sigma-1)(\boldsymbol{\Upsilon} \cdot \mathbf{k})+\sigma \Upsilon_{x}\right)=\frac{\sigma}{\mu} f_{y} .
\end{aligned}
$$


The rotational equations are

$$
\begin{aligned}
& \dot{\Upsilon}_{x}=\frac{1}{\sigma\left(1-\mu\left(e^{\star}\right)^{2}\right)}\left(-\mu\left(e^{\star}\right)^{2} \sigma(\mathbf{\Upsilon} \cdot \mathbf{k}) \dot{l}-e^{\star} \sigma f_{z} m+\sigma\left(e^{\star} n+1\right) f_{y}-\mu\left(e^{\star}\right)^{2} \sigma\left(-m f_{x}+l f_{y}\right) l\right), \\
& \dot{\Upsilon}_{y}=\frac{1}{\sigma\left(1-\mu\left(e^{\star}\right)^{2}\right)}\left(-\mu\left(e^{\star}\right)^{2} \sigma(\mathbf{\Upsilon} \cdot \mathbf{k}) \dot{m}+e^{\star} \sigma f_{z} l-\sigma\left(e^{\star} n+1\right) f_{x}-\mu\left(e^{\star}\right)^{2} \sigma\left(-m f_{x}+l f_{y}\right) m\right), \\
& \dot{\Upsilon}_{z}=\frac{1}{\sigma\left(1-\mu\left(e^{\star}\right)^{2}\right)}\left(-\mu\left(e^{\star}\right)^{2} \sigma(\mathbf{\Upsilon} \cdot \mathbf{k}) \dot{n}-e^{\star} \sigma\left(-m f_{x}+l f_{y}\right)\left(1+\mu e^{\star} n\right)\right) .
\end{aligned}
$$

Hurwitz coefficients. The values of the parameters in the polynomial

$$
r_{0} z^{4}+r_{1} z^{3}+r_{2} z^{2}+r_{3} z+a_{4}=0
$$

where $z=\frac{n+1}{1-n}$, are

$$
\begin{aligned}
r_{0}= & \left(1+e^{\star}\right)^{2} \sigma^{2}\left(1+\operatorname{Fr}^{-1} e^{\star}\left(1+e^{\star}\right)^{2} \mu-\sigma-e^{\star}\left(e^{\star} \mu+\sigma\right)\right), \\
r_{1}= & -2\left(1+e^{\star}\right)^{2} \sigma\left(2 \operatorname{Fr}^{-1} e^{\star} \mu\left(-2+\left(e^{\star}\right)^{2}(2 \mu-\sigma)+\sigma\right)+\sigma\left(-1+\left(e^{\star}\right)^{2} \mu+\sigma+2 e^{\star} \sigma\right)\right), \\
r_{2}= & 2 e^{\star}\left(-3\left(1+e^{\star}\right)^{2} \sigma^{3}+\operatorname{Fr}^{-1} \mu\left(8\left(-1+\left(e^{\star}\right)^{2} \mu\right)^{2}\right.\right. \\
& \left.\left.-8\left(-1+\left(e^{\star}\right)^{2}\right)\left(-1+\left(e^{\star}\right)^{2} \mu\right) \sigma+3\left(-1+\left(e^{\star}\right)^{2}\right)^{2} \sigma^{2}\right)\right), \\
r_{3}= & -2 \sigma\left(2 \operatorname{Fr}^{-1}\left(-1+e^{\star}\right)^{2} e^{\star} \mu\left(-2+\left(e^{\star}\right)^{2}(2 \mu-\sigma)+\sigma\right)-\left(1+e^{\star}\right)^{2} \sigma\left(-1+\left(e^{\star}\right)^{2} \mu+\sigma-2 e^{\star} \sigma\right)\right), \\
r_{4}= & \sigma^{2}\left(-1+\sigma+e^{\star}\left(-2+\operatorname{Fr}^{-1} \mu+\sigma+e^{\star}\left(-1+\left(\left(1+e^{\star}\right)^{2}\right.\right.\right.\right. \\
& \left.\left.\left.\left.+\operatorname{Fr}^{-1}\left(-2+e^{\star}\right)\left(2+\left(-2+e^{\star}\right) e^{\star}\right)\right) \mu-\sigma-e^{\star} \sigma\right)\right)\right) .
\end{aligned}
$$

Acknowledgment. We wish to acknowledge Andy Ruina for helpful comments.

\section{REFERENCES}

[1] A. M. Bloch, P. S. Krishnaprasad, J. E. Marsden, and T. S. Ratiu, Dissipation induced instabilities, Ann. Inst. H. Poincaré Anal. Non Linéaire, 11 (1994), pp. 37-90.

[2] A. M. Bloch, P. S. Krishnaprasad, J. E. Marsden, and T. S. Ratiu, The Euler-Poincaré equations and double bracket dissipation, Comm. Math. Phys., 175 (1996), pp. 1-42.

[3] N. M. Bou-Rabee, J. E. Marsden, And L. A. Romero, A geometric treatment of Jellett's egg, ZAMM, submitted, 2004.

[4] M. G. Clerc and J. E. Marsden, Dissipation-induced instabilities in an optical cavity laser: A mechanical analog near the 1:1 resonance, Phys. Rev. E, 64 (2001), 067603.

[5] R. J. Cohen, The tippe top revisited, Amer. J. Phys., 45(1) (1977), pp. 12-17.

[6] S. EBenfeld And F. SCHECK, A new analysis of the tippe top: Asymptotic states and Liapunov stability, Ann. Phys., 243 (1995), pp. 195-217.

[7] F. R. Gantmacher, The Theory of Matrices, Vol. II, Chelsea, New York, 1959.

[8] H. K. KhaliL, Nonlinear Systems, 3rd ed., Prentice-Hall, Upper Saddle River, NJ, 2002, pp. 126-133.

[9] J. E. Marsden And T. S. Ratiu, Introduction to Mechanics and Symmetry, 2nd ed., Springer-Verlag, New York, 1999.

[10] H. K. Moffatt And Y. Shimomura, Classical dynamics: Spinning eggs-a paradox resolved, Nature, 416 (2002), pp. 385-386.

[11] A. C. OR, The dynamics of a tippe top, SIAM J. Appl. Math., 54 (1994), pp. 597-609.

[12] E. J. Routh, The Advanced Part of a Treatise on the Dynamics of a System of Rigid Bodies, Macmillan, New York, 1905.

[13] A. Ruina, Rolling and Sliding of Spinning Things: Euler's Disk, Jellett's Egg and Moffatt's Nature. http://tam.cornell.edu/ ruina/hplab/Rolling\%20and\%20sliding/index.html (2002).

[14] W. Thomson and P. G. TAit, Treatise on Natural Philosophy, Cambridge University Press, Cambridge, UK, 1879. 